\title{
Efecto del V y el Si Sobre la Microestructura de Depósitos Realizados con Electrodos Tubulares Revestidos de Alto Contenido de Mn (Hadfield)
}

\author{
Manuel Rodríguez ${ }^{1}$, Lorenzo Perdomo¹, Luis Béjar², José A. Moreno², Ariosto Medida³, Juan F. Soriano², Ismeli Alfonso ${ }^{4}$ \\ 1 Universidad Central "Marta Abreu” de Las Villas - UCLV, Centro de Investigaciones de Soldadura, Santa Clara, Villa Clara, Cuba. \\ 2 Universidad Michoacana de San Nicolás de Hidalgo - UMICH, Facultad de Ingeniería Mecánica, Morelia, Michoacán, México. \\ 3 Universidad Michoacana de San Nicolás de Hidalgo - UMICH, Instituto de Investigaciones en Metalurgia y Materiales, Morelia, \\ Michoacán, México. \\ 4 Universidad Nacional Autónoma de México - UNAM, Instituto de Investigaciones en Materiales, Unidad Morelia, Morelia, \\ Michoacán, México.
}

Recibido: 01 Jun., 2017

Aprobado: 30 Ago., 2017

E-mails: manuelr@uclv.edu.cu (MR), Iperdomo@uclv.edu.cu (LP), Ibejargomez@yahoo.com.mx (LB), juanfsoriano@me.com (JAM), ariosto@umich.mx (AM), ialfonso@unam.mx (IA)
Resumen: Las aleaciones para el relleno superficial Fe-Mn-C (Hadfield) han demostrado una excelente resistencia al desgaste bajo altas cargas dinámicas. En los últimos años se han realizado numerosos estudios para aumentar la resistencia al desgaste y mejorar su desempeño, a través de la introducción de otros elementos de aleación. En el presente trabajo se investiga la microestructura y dureza de los depósitos de relleno con alto contenido de $\mathrm{Mn}$ y adiciones de $1.2 \%$ de $V$ y $2.4 \%$ de Si. Los depósitos estudiados se realizaron utilizando electrodos tubulares revestidos fabricados a escala de laboratorio. Las fases y microconstituyentes en el metal depositado se identificaron mediante microscopía óptica (MO), electrónica de barrido (MEB), difracción de rayos $X$ (DRX), dureza y microdureza. De acuerdo a los resultados obtenidos, la adición de $V$ al sistema aleante originó que la fase predominante fuera la austenita. Además, contribuyó a la formación de carburos de vanadio (VC) en la microestructura de la capa de relleno, sin observarse la presencia de carburos complejos. Por otra parte, la presencia de Si favoreció la formación de una red de ferrita interdendrítica. La adición de estos elementos de aleación mejoró las propiedades de estos depósitos, potencializando su uso en aplicaciones que requieren alta resistencia al desgaste bajo altas cargas.

Palabras-clave: Hadfield; Microestructura; Relleno; Vanadio; Silicio.

\section{Effect of $\mathrm{V}$ and $\mathrm{Si}$ on the Microstructure of Deposits Welded Using Tubular Coated Electrodes with High Mn Content (Hadfield)}

\begin{abstract}
Fe-Mn-C (Hadfield) alloys for surfacing weld deposits have demonstrated excellent wear resistance under high dynamic loads. In recent years, numerous studies have been conducted to improve their wear resistance and performance through the introduction of others alloying elements. In the present work, microstructure and hardness of high Mn surfacing welds with 1.2\% V and $2.4 \% \mathrm{Si}$ additions were investigated. The surfacing welds were deposited using experimental tubular coated electrodes. Phases and micro constituents in the resulting deposits were identified using optical microscopy (OM), scanning electron microscopy (SEM), X-ray diffraction (XRD), hardness and micro hardness. Based on the obtained results, $V$ additions to the alloy led to the formation of austenite as predominant phase. Besides, contributed to the formation of vanadium carbides (VC), without the presence of complex carbides. Otherwise, Si addition favored the formation of an interdendritical ferrite network. The addition of these elements favored the increase in the properties of the weld deposits, potentializing their use in applications that require high wear resistance under high loads.
\end{abstract}

Key-words: Hadfield; Microestructure; Surfacing; Vanadium; Silicon.

\section{Introducción}

El primer acero fundido austenítico al Mn destinado a garantizar una buena resistencia al desgaste por fatiga superficial sin perder su tenacidad, fue desarrollado por primera vez en Inglaterra en el año 1883 por Robert Hadfield. Esta aleación en su variante inicial tenía una composición de $1.2 \%$ de $\mathrm{C}$ y $12 \%$ de $\mathrm{Mn}$ [1,2], pero en la actualidad existen diversas composiciones de aleaciones fundidas del tipo Hadfield que emplean otras proporciones de $\mathrm{C}$ y $\mathrm{Mn}$, e incluso se le han incorporado nuevos elementos de aleación tales como Si, $\mathrm{Cr}, \mathrm{Ni}, \mathrm{Mo}$, etc. Estas adiciones buscan mejorar las propiedades de las aleaciones, como 
se reporta en la norma ASTM-A128 [3]. En estas aleaciones, el Mn actúa como estabilizador de la fase austenítica, retrasando su transformación isotérmica para un contenido de $\mathrm{C}$ de hasta 1.2\%. Para contenidos superiores de $\mathrm{C}$, es posible la precipitación de carburos $(\mathrm{Fe}, \mathrm{Mn})_{3} \mathrm{C}$ en los límites de grano, que pueden originar una disminución en la tenacidad, lo cual es perjudicial en piezas de dimensiones considerables que estén sujetas a impactos severos [2,4]. El endurecimiento de estos aceros en servicio es atribuido a una serie de teorías, siendo algunas diferentes en cuanto a los fenómenos que explican. La más aceptada, plantea que el buen comportamiento al desgaste por fatiga superficial de los aceros Hadfield se debe a su capacidad de endurecimiento por la deformación originada por las condiciones de trabajo de los elementos de maquinaria sometidos a impactos. Este proceso de endurecimiento es consecuencia de la interacción de las dislocaciones y la concentración de átomos intersticiales conocido como interacción Cottrell-Bilby [5]. Esta hipótesis es coherente con la reportada por Iglesias y Schuz [6], donde se plantea que el endurecimiento se debe a la presencia de elementos intersticiales relacionados con el movimiento de las dislocaciones por deslizamiento y maclaje. Respecto al efecto de los elementos de aleación en estas aleaciones, se reporta en la literatura que contenidos de Si de entre 1 y $2 \%$ incrementan el límite elástico. No obstante, cuando se superan estos valores se reduce la solubilidad del $\mathrm{C}$ en la austenita y por lo tanto su estabilidad a temperatura ambiente [1]. De la misma manera que el $\mathrm{Si}$, el $\mathrm{Cr}$ puede aumentar el límite elástico de la aleación, pero para contenidos superiores al $2.5 \%$ genera carburos insolubles que fragilizan la estructura. La adición al sistema aleante de Mo puede mejorar la tenacidad y favorece la reducción de las grietas en caliente, mientras que el $\mathrm{Ni}$ estabiliza la austenita y limita la precipitación de carburos [1]. Por otra parte, el V se caracteriza por ser un fuerte formador de carburos, y su presencia en los aceros al Mn garantiza un incremento a la resistencia a la fluencia, por lo que disminuye la ductilidad de estas aleaciones. Cuando este elemento es añadido en un rango de $0.5-2 \%$ se recomienda realizar tratamientos térmicos de austenitización seguido de un envejecimiento. Esto permite alcanzar esfuerzos de fluencia superiores a los $700 \mathrm{MPa}$, lo que amplía sus aplicaciones [7]. En relación al empleo del $V$ en las aleaciones Hadfield, se reportan trabajos donde se analiza su efecto en la microestructura de aceros fundidos [8,9]. Méndez et al. [8], por ejemplo, evalúan el comportamiento del acero Hadfield con contenidos de $\checkmark$ entre 1-2\%, revestidos con varios electrodos al $\mathrm{Mn}$ y $\mathrm{Cr}$, comprobando la elevada resistencia a la deformación que adquiere el metal del depósito, pero con una baja capacidad de endurecimiento por deformación. Por otra parte, Sant y Smith [9] realizaron un estudio con los mismos aceros, pero analizando el comportamiento de las fundiciones en cuanto al mecanismo de endurecimiento por deformación con la adición de $\mathrm{V}$ y encontrando el efecto favorable de este elemento en cuanto a la resistencia al desgaste. Al utilizar mayores contenidos de V (5-8\%) en las fundiciones de acero tipo Hadfield [9], en la microestructura aparece una red de carburos con una dureza de $2600 \mathrm{HV}$, lo que incrementa en este caso la resistencia al desgaste por abrasión, pero reduce su capacidad de trabajar en presencia de impactos severos.

Para lograr depósitos totalmente austeníticos, durante la aplicación de recubrimientos superficiales utilizando consumibles de soldadura que aporten aleaciones Hadfield Fe-Mn-C, hay que tener en cuenta la velocidad de enfriamiento de la capa aportada [10,11]. Esto se debe al calentamiento del metal base, originando que la capa de relleno permanezca un tiempo apreciable a temperaturas superiores a los $400^{\circ} \mathrm{C}$, y una vez enfriada se obtiene una microestructura totalmente heterogénea formada por carburos $(\mathrm{Fe}, \mathrm{Mn})_{3} \mathrm{C}$, que se sitúan en el límite de los granos de la austenita y la solución sólida $\alpha$ sobresaturada de $C$ (martensita). En este estado microestructural es prácticamente imposible que el acero se endurezca por deformación plástica, debido a que las fases secundarias (carburos) funcionan como barreras impidiendo el movimiento de las dislocaciones. La presencia perjudicial de estas fases se puede revertir mediante el tratamiento térmico de austenizado [11]. La aplicación de este tratamiento térmico hace que los costos de los trabajos de relleno superficial se incrementen notablemente. En ocasiones la solución se basa en sumergir la pieza a rellenar en agua, para de esta manera evitar el enfriamiento lento y la aparición de carburos, aunque existe el peligro de la formación de martensita.

En relación a los consumibles que depositan aceros Hadfield, para el recubrimiento superficial en la literatura se encontraron trabajos $[10,12]$ que evalúan diversas composiciones de electrodos al $\mathrm{Mn}$, con la adición de alguno de los elementos anteriormente señalados, como el $\mathrm{Ni}$ y $\mathrm{Cr}$. En cuanto al $\mathrm{V}$, hasta ahora no se han encontrado trabajos sobre su utilización en la fabricación de electrodos de este tipo. La base del presente trabajo es la adición de este elemento, ya que su presencia puede ser favorable en el sistema de aleación ya que favorece la formación de carburos, incluso para bajos contenido de este elemento [13,14]. Su presencia en los recubrimientos del tipo Hadfield puede evitar la formación de otros microconstituyentes (carburos complejos) ricos en Mn, lo cual origina que la cantidad de asutenita disminuya, incluso aumentando el contenido de martensita. Además, como se ha 
Efecto del V y el Si Sobre la Microestructura de Depósitos Realizados con Electrodos Tubulares Revestidos de Alto Contenido de Mn (Hadfield)

planteado, añadiendo $\mathrm{V}$ se pueden formar carburos del tipo $\mathrm{V}_{\mathrm{n}} \mathrm{C}_{\mathrm{m}}$ en lugar de carburos complejos del tipo $\mathrm{Me}_{3} \mathrm{C}$ en los límites del grano austenítico. De esta forma no se limita la capacidad de endurecimiento de esta fase y se mantiene la efectividad de estos recubrimientos superficiales. A pesar de que desde este punto de vista un depósito totalmente austenítico es conveniente, esta fase con altos contenidos de $\mathrm{Mn}$ en los aceros Hadfield es muy susceptible al fenómeno de fragilidad intergranular, siendo la causa principal de las fallas en servicio e incluso en el propio proceso de relleno superficial [2]. En este sentido, la presencia de determinadas cantidades de Si puede facilitar la presencia de ferrita interdendrítica, evitando el agrietamiento y como resultado mayor adherencia y tenacidad de la capa de relleno [2]. Se establece que es posible añadir hasta $2.2 \%$ de Si en las aleaciones austeníticas al $\mathrm{Mn}$, lo cual puede incrementar su tenacidad [15]. Con valores superiores de este elemento existe el riesgo de que se pueda afectar la estabilidad de la austenita a temperatura ambiente, aunque no se conoce el resultado si en el sistema de aleación está presente el V. En correspondencia a lo anteriormente señalado, en el presente trabajo se añadirán al sistema Fe-Mn-C tanto $\mathrm{V}$ como $\mathrm{Si}$, con la finalidad de estudiar el efecto complementario de estos dos elementos sobre la microestructura, fundamentalmente elevando la cantidad de Si por encima del valor utilizado en las piezas fundidas. El objetivo final de estas modificaciones es aumentar la efectividad del relleno superficial mediante los procesos de soldadura con consumibles basados en dicha aleación.

\section{Materiales y Métodos}

Para la realización de los depósitos superficiales del sistema Fe-Mn-C-V-Si, en el presente trabajo se utilizaron electrodos fabricados en el Centro de Investigaciones en Soldadura, de la Universidad Central de las Villas, Cuba. Este tipo de consumible se fabricó a escala de laboratorio con un diámetro de $4 \mathrm{~mm}$, cuyas características principales se observan en la Figura 1, donde también se muestra el alambre empleado como núcleo.

El espesor del revestimiento fue de $1.5 \mathrm{~mm}$, y se utilizaron los minerales que se muestran en la Tabla 1, con las proporciones indicadas. Estos minerales se mezclaron en una proporción 100:30 con silicato de sodio, que fue utilizado como aglutinante.

La composición química de los depósitos obtenidos utilizando los electrodos tubulares revestidos aparece en la Tabla 2. Estos valores son los obtenidos en la última capa, donde no hay dilución, tomando en cuenta que es un revestimiento con multipasadas. El uso de un electrodo tubular garantiza que el revestimiento contenga el porcentaje de $\mathrm{C}$ necesario, ya que por el contenido de $\mathrm{C}$ en las ferroaleaciones utilizadas para aportar $\mathrm{Mn}$ y $\mathrm{V}$, no era posible lograr el $\mathrm{C}$ necesario solo a través de la carga. Por otro lado, este consumible cuenta con un revestimiento
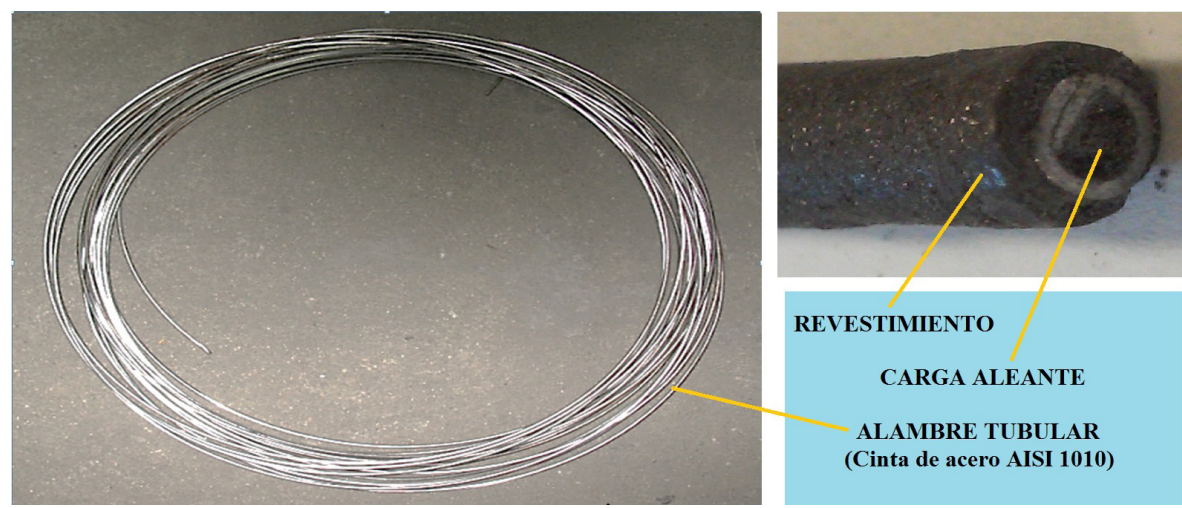

Figura 1. Alambre y electrodo fabricado para la realización de los depósitos.

Tabla 1. Composición química del revestimiento utilizado en los electrodos.

\begin{tabular}{cc}
\hline Componente & Proporción (\%) \\
Calcita & 50 \\
Fluorita & 20 \\
Rutilo & 12 \\
Grafito & 18 \\
\hline
\end{tabular}


Tabla 2. Composición química del depósito realizado con los electrodos experimentales.

\begin{tabular}{ccccccc}
\hline & \multicolumn{5}{c}{ Composición del depósito (\%) } \\
\cline { 2 - 7 } Electrodo & $\mathbf{C}$ & Mn & V & Si & Fe \\
EFC - H (experimental) & 1.1 & 12.3 & 1.2 & 2.4 & Balance \\
\hline
\end{tabular}

que garantiza una buena estabilidad del arco con bajas intensidades de corriente, lo cual permite que el aporte térmico sea bajo en comparación al FCAW. Si el aporte térmico es bajo se reduce la posibilidad de precipitación de carburos complejos con Mn, aspecto importante en los recubrimientos del tipo Hadfield.

Los depósitos se realizaron mediante soldadura manual por arco eléctrico sobre chapas de acero AISI/SAE 1020 (250X100X10) en varias capas, como se observa en la Figura 2a, mostrándose igualmente la ubicación de la zona de estudio. Los parámetros de soldadura se indican en la Tabla 3. Durante la operación se evitó el enfriamiento rápido de la aleación con el fin de reproducir en lo posible las condiciones reales en las cuales se aplica el recubrimiento superficial en la industria. Por esta razón se realizó el depósito de forma continua, con temperaturas entre pasadas de $100-120^{\circ} \mathrm{C}$, finalmente enfriando al aire. En la Figura $2 \mathrm{~b}$ igualmente se muestra un ejemplo de un recubrimiento depositado bajo las condiciones anteriores, observándose la similitud con la Figura 2a. Es de destacar la excelente operatividad del consumible, pues el revestimiento facilitó el encendido y estabilidad del arco favorecido por la calcita, rutilo y el propio grafito, que tienen bajos potenciales de ionización (Ca, Ti y C).
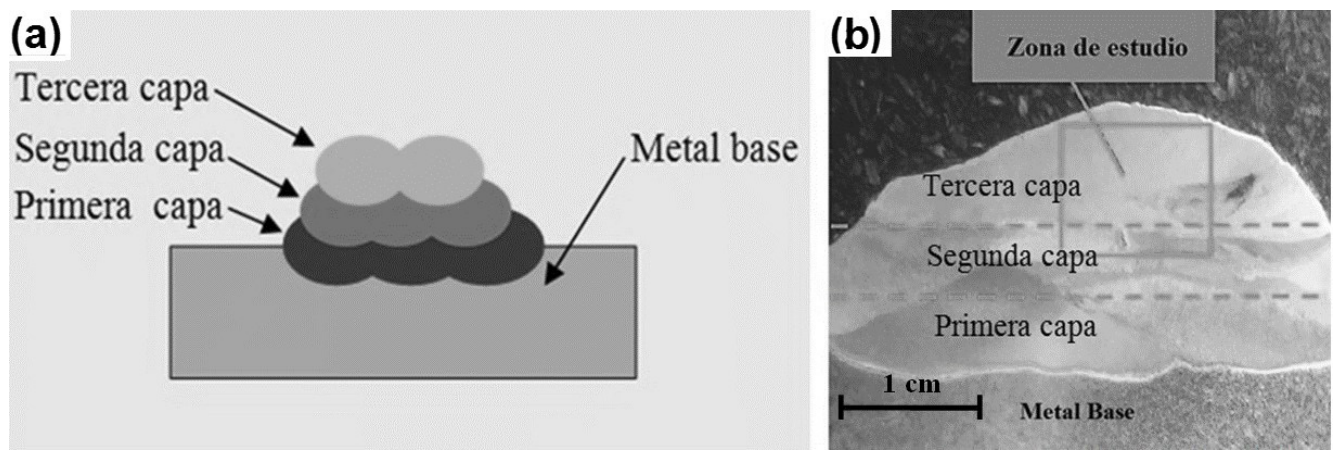

Figura 2. (a) Esquema de realización de los depósitos de soldadura, (b) Macrografía del depósito de relleno.

Tabla 3. Parámetros de soldadura utilizados para la realización de los depósitos.

\begin{tabular}{cccc}
\hline $\begin{array}{c}\text { Intensidad } \\
\text { de corriente (A) }\end{array}$ & Voltaje de arco (V) & $\begin{array}{c}\text { Velocidad de } \\
\left.\text { soldadura (cm.min }{ }^{-1}\right)\end{array}$ & $\begin{array}{c}\text { Tipo de Corriente y } \\
\text { Polaridad }\end{array}$ \\
\hline $110-120$ & $23-25$ & $20-23$ & CD $^{+}$ \\
\hline
\end{tabular}

Para la caracterización metalográfica el depósito fue cortado transversalmente en secciones de $20 \mathrm{~mm}$, utilizando refrigerante para evitar el sobrecalentamiento de la aleación. La superficie a estudiar se preparó empleando técnicas de pulido convencionales. Para revelar las estructuras se utilizó una solución de Nital al $5 \%$ durante 15 segundos. El análisis de la microestructura se realizó utilizando un Microscopio Óptico LABMED MET 400, y un Microscopio Electrónico de Barrido MEB marca JEOL JSM 7600F, el cual cuenta con un detector de Espectroscopía de Energía Dispersiva (EDX) Bruker XFlash6130. El estudio se complementó utilizando difracción de rayos $\mathrm{X}$ mediante un equipo marca Bruker D-8 Advance con radiación CuKa operado a 30kV y 25mA. Por último, para el estudio de la microdureza de las fases presentes, se empleó un equipo Shimadzu HMV-2 con una carga de 4.9 N. Para determinar el contenido de las fases se utilizó análisis de imágenes mediante el programa SigmaScanPro 5.0.

\section{Resultados y Discusión}

En la Figura 3 se muestra la microestructura obtenida por microscopía óptica para el depósito de soldadura realizado de acuerdo a los parámetros anteriormente descritos. Como puede observarse, se obtuvo una microestructura mayoritariamente austenítica de forma detrítica con la presencia. El contenido de estas fases, obtenido mediante 


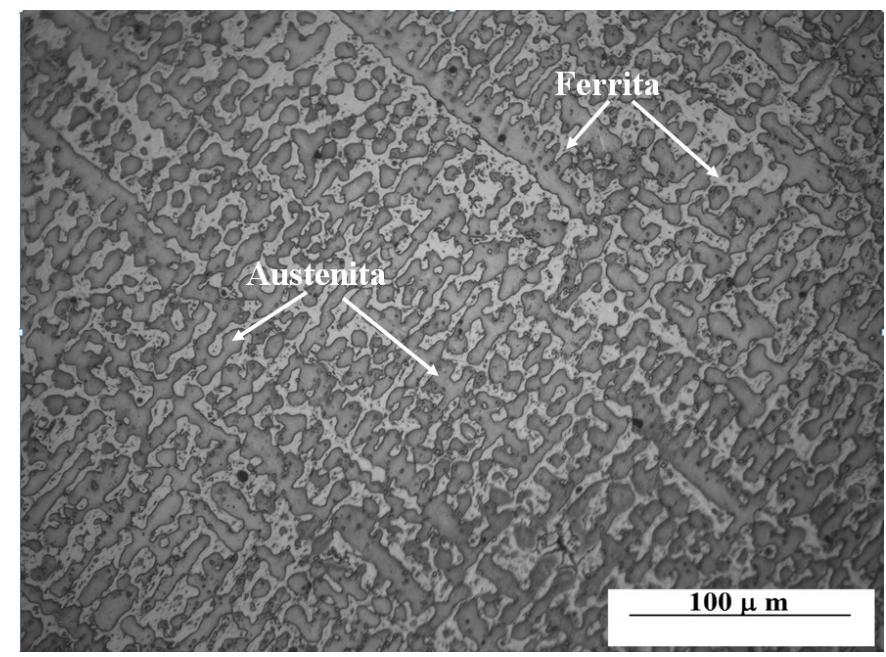

Figura 3. Microestructura de los depósitos donde se observa la presencia de dos fases.

análisis de imágenes, mostró que el $72 \%$ corresponde a la fase austenítica, mientras que el porcentaje de la ferrita interdentrítica fue de $28 \%$. La presencia de fases de menor tamaño dentro de estas dos fases principales, como podrían ser carburos, se analizará más adelante mediante estudios a mayores magnificaciones.

En la Figura 4 se puede apreciar el difractograma obtenido mediante DRX para el depósito realizado con el electrodo experimental. Como puede observarse se confirma que las fases predominantes son la austenita y la ferrita. Los picos de austenita fueron más intensos que los de ferrita, indicando un mayor contenido de esta fase, lo cual está en correspondencia con lo observado mediante microscopía. Asimismo, se puede apreciar la presencia de carburos VC y de óxido de hierro.

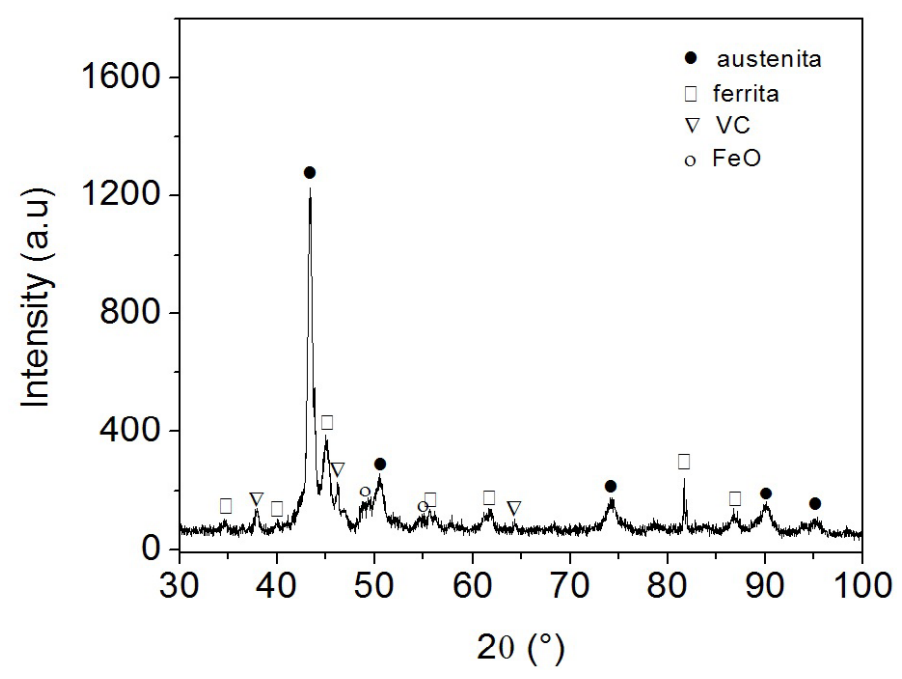

Figura 4. Difractograma obtenido mediante difracción de rayos-X del depósito, donde se aprecian las fases presentes.

Para fundamentar los resultados anteriormente mostrados, es importante revisar la evolución de las fases desde el estado líquido hasta el enfriamiento. Este estudio permitirá analizar el efecto que han tenido los elementos de aleación sobre la microestructura final obtenida. En la literatura [15] se representa el diagrama de equilibrio para las fases presentes de una aleación perteneciente al sistema Fe- C-Mn (ver Figura 5, para contenidos de carbono hasta $1.6 \%$ en los intervalos de temperatura comprendidos entre $400{ }^{\circ} \mathrm{C}$ y $1000^{\circ} \mathrm{C}$ ). En este diagrama se pueden diferenciar una zona trifásica a temperatura de $400{ }^{\circ} \mathrm{C}$, compuesta por las fases $\alpha+\gamma+M_{3} \mathrm{C}$, para contenidos de $\mathrm{C}$ en el orden de 1.2\%. Según lo observado en la Figura 3, donde se mostró la microestructura correspondiente a los depósitos obtenidos, se formó austenita y ferrita, lo cual no se corresponde con las fases 


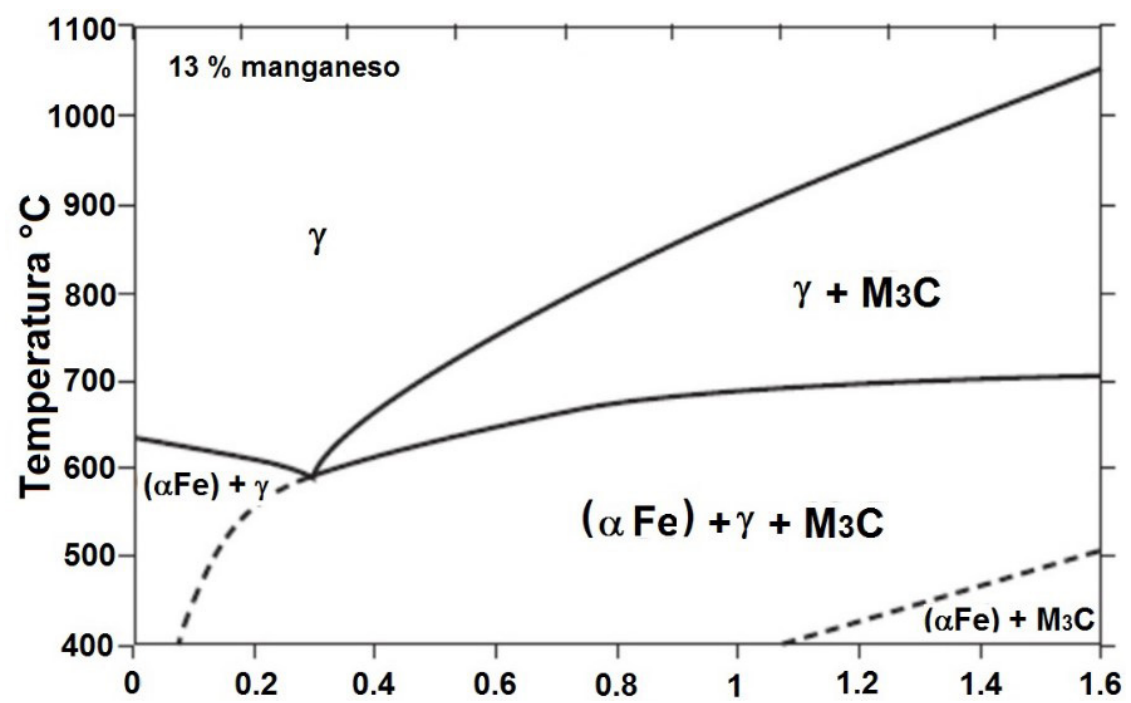

Figura 5. Diagrama de equilibrio para las aleaciones Hadfield con $13 \%$ de Mn y hasta $1.6 \%$ de C [modificado de 15].

esperadas según el diagrama de equilibrio. Las causas de esta diferencia están relacionadas en este caso con dos aspectos fundamentales: i) que la aleación depositada no es el acero Hadfield convencional a base de $\mathrm{C}$ y $\mathrm{Mn}$, ya que ha tenido modificaciones con la adición de $\mathrm{V}$ y Si; y ii) que en la capa de relleno analizada las fases que se presentan están determinadas por velocidades de enfriamiento propias del recubrimiento superficial.

Con la finalidad de complementar el análisis de las fases presentes, y analizar la morfología de los carburos, se tomaron imágenes a mayores magnificaciones. En las Figuras $6 a$ y $6 b$, tomada mediante MEB utilizando electrones retrodispersados y secundarios, respectivamente, se destaca que además de las fases austenita y ferrita se encuentran carburos de vanadio de tamaño relativamente pequeños (1-2 $\mu \mathrm{m})$. Estos carburos son de forma irregular y están distribuidos uniformemente por la estructura, en su mayoría localizados en los límites del grano austenítico. El porcentaje de estos carburos en la microestructura es relativamente bajo (5.6\%), no obstante pueden tener cierta influencia en la dureza, y por ende sobre la resistencia al desgaste abrasivo del metal depositado. Por otra parte, estos carburos no limitarían al endurecimiento de la austenita por deformación plástica. Una vez redefinido el contenido de las fases debido a la presencia de los VC, los porcentajes serían $28 \%$ Ferrita $+66.4 \%$ Austenita + 5.6\% VC.

La presencia de los carburos anteriormente descritos se confirma en el análisis mediante EDX, mostrado en la Figura 7, la cual presenta un mapeo de los elementos fundamentales para el presente estudio. Como puede apreciarse, el V y el C están distribuidos fundamentalmente en los carburos (círculo). Asimismo, se muestra el estudio puntual mediante EDX de uno de estos carburos, siendo los picos más intensos los correspondientes al C y el V. En este análisis también se aprecia que el Mn se concentra principalmente en la fase austenita, sin
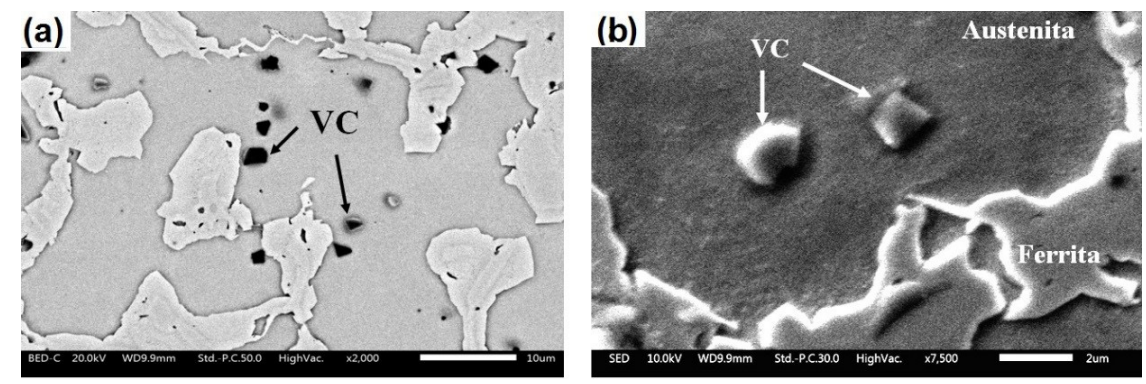

Figura 6. (a) Microestructura obtenida mediante MEB con electrones retrodispersados del depósito donde se aprecian la austenita, la ferrita, y carburos de vanadio, VC (señalados con flechas); (b) Microestructura a mayor magnificación obtenida mediante MEB con electrones secundarios donde se aprecia la forma de los carburos de vanadio, VC. 
Efecto del V y el Si Sobre la Microestructura de Depósitos Realizados con Electrodos Tubulares Revestidos de Alto Contenido de Mn (Hadfield)
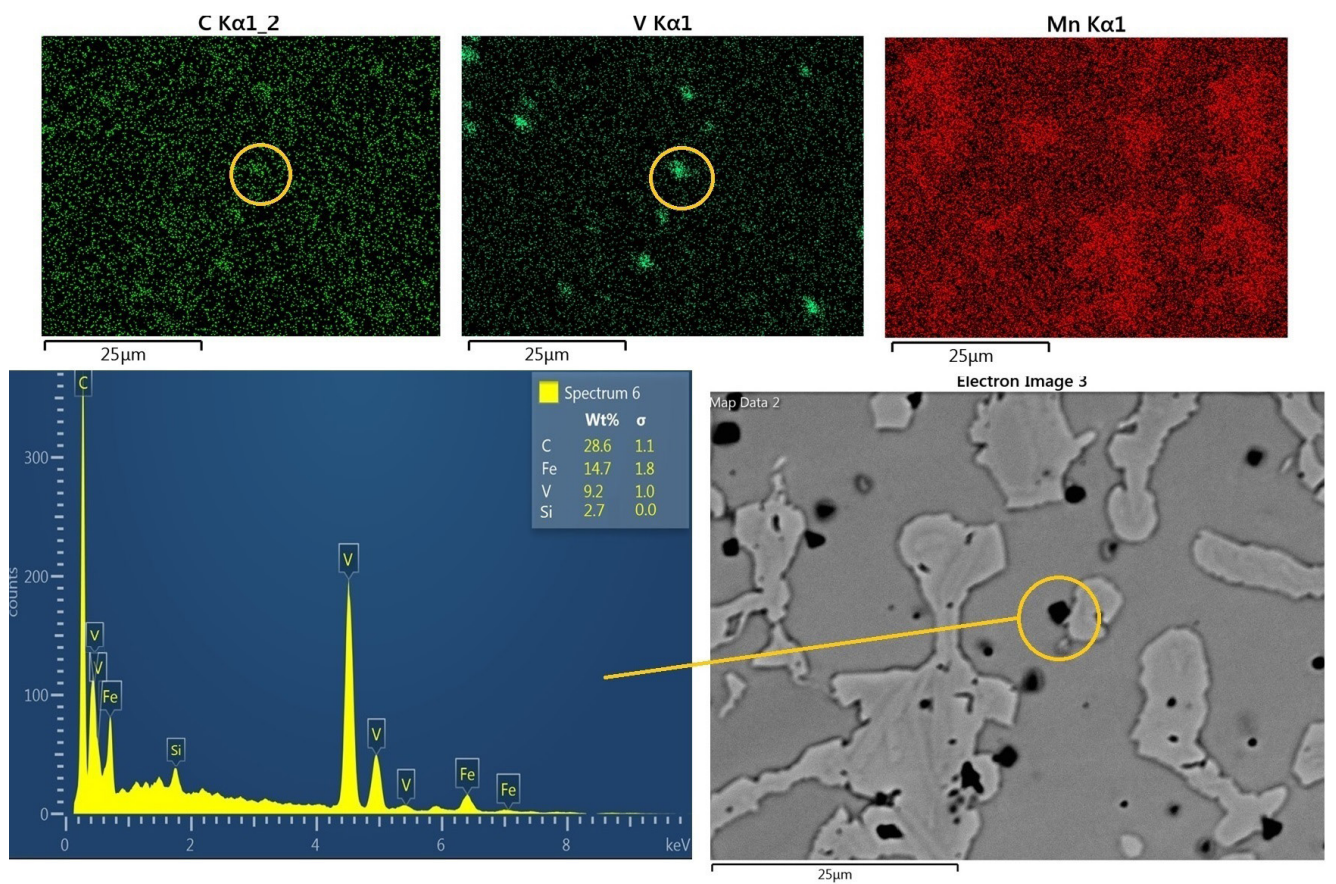

Figura 7. Mapeo por elementos mediante EDX del depósito de soldadura, donde se muestra la distribución de los elementos químicos principales y EDX puntual de uno de los carburos VC.

evidencias de la presencia de carburos $(\mathrm{Fe}, \mathrm{Mn})_{3} \mathrm{C}$ o de martensita. Este resultado está estrechamente relacionado con la presencia del $\mathrm{V}$ y su alta afinidad por el $\mathrm{C}$ para formar carburos [13,14,16], lo cual reduce las posibilidades de la precipitación del carburo $(\mathrm{Fe}, \mathrm{Mn})_{3} \mathrm{C}$. Por esta razón el $\mathrm{V}$ también evita la difusión del $\mathrm{Mn}$ a través de la red cristalina, lo cual garantiza que perduren zonas en la microestructura con niveles apreciables de este elemento. La presencia del Mn en solución sólida $y$ hasta el enfriamiento del depósito garantiza la permanencia de austenita a temperatura ambiente, debido a que se retardaría la transformación isotérmica de la austenita [17]. Como se ha explicado, la presencia de la austenita una vez realizado el relleno superficial es muy importante, al ser la fase que proporciona a los recubrimientos del tipo Hadfield la capacidad de endurecimiento por deformación. Es de destacar que el Si se encontró uniformemente distribuido en toda la microestructura, razón por la cual no se presentó en el mapeo elemental.

Los resultados de dureza para los depósitos, y de microdureza para las fases presentes en ellos, se muestran en la Tabla 4. Como puede apreciarse, la dureza promedio del depósito es de $33 \mathrm{HRC}$, lo cual es un valor que se corresponde con los depósitos de este tipo de aleación [6]. En cuanto a la microdureza, se puede apreciar que para el caso de la austenita los valores son mucho mayores, alcanzando $307 \mathrm{HV}$, mientras que la ferrita solo llega a $232 \mathrm{HV}$. Estos valores se corresponden con los valores convencionales para estas fases, específicamente en los aceros austeníticos al Mn [15].

Tabla 4. Dureza y microdureza del depósito.

\begin{tabular}{ccccc}
\hline \multirow{2}{*}{ Mediciones } & Dureza & \multicolumn{2}{c}{ Microdureza } \\
\cline { 2 - 3 } \cline { 4 - 5 } \cline { 4 - 5 } & HRC & & Austenita (HV) & Ferrita (HV) \\
2 & 34 & 311 & 241 \\
3 & 31 & 316 & 232 \\
4 & 36 & 309 & 237 \\
5 & 32 & 296 & 225 \\
Promedio & 32 & 305 & 226 \\
& 33 & 307 & 232 \\
\hline
\end{tabular}


El Si por su parte, es un elemento que incrementa sustancialmente la estabilidad de la ferrita para concentraciones de 1-2\% de C [18]. Este efecto del Si presente en la zona interdendrítica, así como la disminución del contenido de $\mathrm{Mn}$ (Figura 7), son consideradas las causas principales para que se forme la fase ferrítica en la microestructura de los depósitos. La ferrita en los recubrimientos superficiales logra incrementar el límite elástico y la tenacidad de la capa de relleno, siendo la causa principal de que los depósitos estudiados estén libres de grietas, tal como se aprecia en la macrografía (Figura 2 b) y micrografía (Figura 3) obtenidas en la investigación. Por otro lado, la ferrita disuelve mayor cantidad de impurezas en su microestructura cristalina que la austenita lo que reduce la posibilidad de eutécticos de baja temperatura de fusión en los límites de grano, causas fundamentales de las grietas de solidificación. Es importante señalar que en los aceros fundidos del tipo Hadfield no es objetivo esencial la presencia de la fase ferrítica, ya que las potencialidades de endurecimiento de los depósitos por deformación plástica pueden reducirse al formarse menos austenita. Sin embargo, en el caso del relleno superficial mediante los procesos de soldadura cierta cantidad de ferrita puede ser favorable desde el punto de vista de la reducción a la sensibilidad al agrietamiento de los depósitos tipo Hadfield. Lo anterior es debido a que se reduce la fragilidad intergranular de la austenita, causada principalmente por la precipitación de carburos en los límites de grano, por la baja capacidad de disolver impurezas de esta fase y por el nivel de tensiones residuales que normalmente persisten en el metal depositado. Todo lo anterior indica que es favorable mantener cierta cantidad de ferrita en los depósitos de la aleación estudiada, con el fin de garantizar la baja sensibilidad al agrietamiento. Estos resultados muestran que es deseable un equilibrio entre la ferrita (por lo anteriormente mencionado), y la austenita (con el fin de conservar las potencialidades de endurecimiento por deformación de esta fase).

\section{Conclusiones}

Una vez analizados los resultados obtenidos al adicionar $1.2 \%$ de $\mathrm{V}$ y $2.4 \%$ de $\mathrm{Si}$ al sistema Fe-Mn-C para el recubrimiento superficial de alto contenido de $\mathrm{Mn}$ (Hadfield), se puede concluir lo siguiente:

- Con la adición de V se favoreció la presencia del Mn en un área significativa de la microestructura y la formación de austenita.

- La adición de V igualmente favoreció la precipitación de VC en lugar de los carburos interdendríticos (Fe, $\mathrm{Mn})_{3} \mathrm{C}$. La formación de VC dispersos no limitaría al endurecimiento de la austenita por deformación plástica.

- La adición de Si y la distribución no uniforme del Mn en la microestructura promovió la formación de un volumen apreciable de ferrita interdendrítica, lo cual reduce la sensibilidad al agrietamiento durante el relleno.

- De las conclusiones anteriores se deriva que la adición combinada de Si y $\mathrm{V}$ originó que los depósitos de relleno tipo Hadfield presenten modificaciones microestructurales que mejorarían sus potencialidades de aplicación relacionadas con la resistencia al desgaste.

\section{Agradecimientos}

Se agradece el apoyo al PREI de la DGAPA de la Universidad Autónoma de México por el apoyo al Dr. Manuel Rodríguez Pérez para realizar la presente investigación, a través del financiamiento para una estancia en el Instituto de Investigaciones de Materiales, Unidad Morelia. Asimismo, se agradece el apoyo técnico de Orlando Hernández Cristóbal y Adriana Tejeda en las caracterizaciones de MEB y DRX, respectivamente.

\section{Referencias}

[1] Heredia JC. Estabilidad de la fase austenítica, variación de la dureza en los aceros al manganeso afectados por el calor y sometidos a impacto. Revista del Instituto de Investigaciones de la Facultad de Geología, Minas, Metalurgia y Ciencias Geográfica. 2012;5(29):99-108.

[2] García A, Varela A, Mier JL. Camba, C, Barbadillo, F. Estudio tribológico de aceros austeníticos tipo Hadfield: Influencia del manganeso en respuesta frente al desgaste. Revista de Metalurgia. 2010;46:47-52.
[3] American Society for Testing and Materials. ASTM A128/ A128M-93: standard specification for steel casting, austenitic Manganese. West Conshohocken: ASTM; 1993.

[4] Subramanyam DK, Swansieger AE. Austenitic manganese steels. In: American Society of Metals. ASM Metal handbook. 10th ed. Illinois: ASTM; 1990. (Vol. 1). p. 822-840.

[5] Lin CY, Wang SW, Chen TM. A study on the wear Behaviour of Hadfield Manganese Steel. Journal of Materials Processing 
Efecto del V y el Si Sobre la Microestructura de Depósitos Realizados con Electrodos Tubulares Revestidos de Alto Contenido de Mn (Hadfield)

Technology. 2002;120(1-3):126-132. http://dx.doi.org/10.1016/ S0924-0136(01)01195-5.

[6] Iglesias C, Schuz E. Aceros austeníticos al manganeso: breve historia. Revista Remetallica. 2004;24(10):9-14.

[7] Giraldo AV. El acero hadfield revisado. Revista Colombiana de Materiales. 2010;3:1-24.

[8] Mendez J, Ghoreshy M, Mackay WBF, Smith TJN, Smith RW. Weldability of austenitic manganese steel. Journal of Materials Processing Technology. 2004;153-154:596-602. http://dx.doi. org/10.1016/j.jmatprotec.2004.04.033.

[9] Sant SB, Smith RW. A study in the work - hardening behavior of austenitic manganese steels. Journal of Materials Science. 1987;22:1808-1814.

[10] Torres Alpizar E, Rodríguez M. Estudio del mecanismo de endurecimiento de los depósitos de soldadura tipo hadfield con alto contenido de Mn. In: VI Simposio Internacional de Soldadura; 1995 Noviembre 15-17; Santa Clara, Cuba; 1995. p. 39-40.

[11] Glownia J, Tezza G, Aslanowicz M, Oscilowski A. Tool cast from steel of composite structure. Archives of metallurgy and Materials. 2013;(58):803-808.

[12] Jankauskas V, Antonov M, Katinas E, Gedzevicius I. Effect of additives on impact - abrasive wear of manual Arc Welded
Hadfield Steel Hardfacing. Journal of Friction and Wear. 2016;37(2):219-227.

[13] Faruk U, Presloscan A, Dukie V. The effect of vanadium content on the properties of $\mathrm{Fe}-\mathrm{C}-\mathrm{Cr}-\mathrm{V}$ white cast iron. Materials and Technology. 2003;37(1-2):19-24.

[14] Wang $\mathrm{Q}$, Xiaoyan $\mathrm{L}$. Effects of $\mathrm{Nb}, \mathrm{V}$ and $\mathrm{W}$ on microstructure and abrasion resistance of Fe-Cr-c hardfacing alloys. Welding Journal. 2010;(Suppl 1):133-139.

[15] Higuera F, Moreno M, Suarez B. Evolución microestructural del acero austenítico al manganeso sometido a tratamiento térmico de temple y revenido. Revistas Ingenierías. 2010; 9 (17):95-206.

[16] Filipovic M, Kamberovic Z, Korac M. Solidification of higt chromium white cast iron with Vanadium. Materials Transactions. 2011;52(3):386-390. http://dx.doi.org/10.2320/matertrans. M2010059.

[17] Higuera O, Tristancho J, Florez L. Fundamentos teóricos de los aceros austeníticos al manganeso. Scientia et Technica. 2007;(34):231-236.

[18] Davis JR. Classification and basic metallurgy of cast irons. In: American Society of Metals. ASM specialty handbook cast iron. Illinois: ASTM; 1996. p. 4-17. 